\title{
An Explosion Aftershock Model with Application to On-Site Inspection
}

\author{
SEAN R. Ford ${ }^{1}$ and Peter LABAK ${ }^{2}$
}

\begin{abstract}
An estimate of aftershock activity due to a theoretical underground nuclear explosion is produced using an aftershock rate model. The model is developed with data from the Nevada National Security Site, formerly known as the Nevada Test Site, and the Semipalatinsk Test Site, which we take to represent soft-rock and hard-rock testing environments, respectively. Estimates of expected magnitude and number of aftershocks are calculated using the models for different testing and inspection scenarios. These estimates can help inform the Seismic Aftershock Monitoring System (SAMS) deployment in a potential Comprehensive Test Ban Treaty On-Site Inspection (OSI), by giving the OSI team a probabilistic assessment of potential aftershocks in the Inspection Area (IA). The aftershock assessment, combined with an estimate of the background seismicity in the IA and an empirically derived map of threshold magnitude for the SAMS network, could aid the OSI team in reporting. We apply the hard-rock model to a M5 event and combine it with the very sensitive detection threshold for OSI sensors to show that tens of events per day are expected up to a month after an explosion measured several kilometers away.
\end{abstract}

Key words: OSI, SAMS, Passive method, Signal processing, Seismic.

\section{Introduction}

An explosion produces an aftershock sequence that is similar in character to a sequence from an earthquake (Kitov and KuzNETsov 1990), so the same Omori decay in time and Gutenberg-Richter distribution in magnitude can be employed to model the explosion aftershock sequence. FORD and WALTER (2010) found that explosion aftershocks were fewer in number and lower in magnitude than earthquake aftershocks for similarly sized explosions and earthquakes. This observation prompted them to use an earthquake aftershock model calibrated from Western US seismicity to test the hypothesis that a given aftershock sequence is due to an earthquake.

\footnotetext{
1 Lawrence Livermore National Laboratory, Livermore, USA. E-mail: sean@1lnl.gov

2 Comprehensive Test Ban Treaty Organization Preparatory Commission, Vienna, Austria.
}

The presence of explosion-induced aftershocks led to the requirement for a seismic capability in an On-Site Inspection (OSI). The search area for an OSI could be greatly reduced if explosion-induced aftershocks could be located. Therefore, it is important to model the spatial and temporal behavior of these aftershocks in order to assess the seismic capability in an OSI. The proposed aftershock rate model can predict this behavior for a given explosion size and location in an OSI-triggering event.

In order to determine the parameters of the aftershock model, we attempted to stack Nevada Test Site (NTS) explosion aftershock sequences, but there were not enough aftershocks detected by the regional networks at the time. We searched for high-resolution studies of explosion aftershock sequences that could provide data on aftershock rate and magnitude distribution. The requirement that magnitude data be available precluded many aftershock studies from use, since most employed an uncalibrated measurement of relative amplitude. This approach is fine for aftershock decay studies, but is not useful for producing an absolute, transportable aftershock model. Three studies were found to fit our requirements, and fortunately, they span a range of environments. In the work that follows, we develop models from each of these studies that we propose can be used as standards in each environment, and then we employ the models to predict aftershock seismicity in the context of OSI planning, deployment, and reporting.

\section{Method}

REASENBERG and Jones $(1989,1994)$ combined the Omori law that describes the power-law decay in the number of aftershocks $N$ with time $t$, 


$$
N(t)=K\left(t-t_{c}\right)^{-p},
$$

for $t_{c}>0$, where $t_{c}$ is the time of the mainshock, $p$ describes the decay, and $K$ is related to the productivity of the sequence, with the Gutenberg-Richter description of magnitude $M$ distribution

$$
N(M)=A 10^{-b M},
$$

where $b$ describes the ratio of small to large events and $A$ is related to the productivity of the sequence, to determine an aftershock rate equation given by

$$
\lambda(t, \Delta M)=10^{a+b \Delta M} t^{-p},
$$

where $\Delta M$ is the difference between the mainshock magnitude $M_{\mathrm{m}}$ and the aftershock magnitude $M$ ( $\left.\Delta M=M_{\mathrm{m}}-M\right)$, and $a$ is proportional to the seismicity rate (WIEMER 2000), which substitutes for the productivity terms, $K$ and $A$, and is given by

$$
a=\log _{10}(K)-b\left(M_{\mathrm{m}}-M_{\min }\right),
$$

where $M_{\min }$ is the magnitude of the smallest event recorded in the aftershock sequence. We calculate the parameters $p, b$, and $K$ for each aftershock study by fitting the distribution of events in time and magnitude and $M_{\mathrm{m}}$ and $M_{\mathrm{min}}$ from the explosion magnitude and minimum recorded aftershock, respectively.

Assuming a nonhomogeneous Poissonian occurrence of aftershocks, the probability of $N$ events occurring above magnitude $M$ within an interval $(0, t)$ is

$$
p(N, t)=\frac{\left(\int_{0}^{t} \lambda(t) d t\right)^{N}}{N !} \exp \left(-\int_{0}^{t} \lambda(t) d t\right) .
$$

FELZER and BRODSKy (2006) showed that a powerlaw decay in aftershock density $\rho$ with radial distance $r$ from the mainshock is appropriate so that aftershock spatial density can be described as

$$
\rho(r)=c r^{-n}
$$

where $n$ is the decay constant and $c$ is related to the number of aftershocks. We calculate the parameter $n$ by fitting the distribution of events in space. The probability density function is from the Pareto distribution of the form

$$
p(r)=\frac{n-1}{r_{\min }}\left(\frac{r}{r_{\min }}\right)^{-n}
$$

where we must assume a minimum distance cutoff $r_{\min }$ where the probability goes to zero at $r<r_{\text {min }}$. The implications of this will be discussed later.

\section{Data and Models}

We make use of three explosion aftershock data sets that encompass a range of geologies. The first is from the well-studied underground nuclear explosion, BENHAM, the second is from the high-explosive underground explosion named the Non-Proliferation Experiment (NPE), and the third is from an underground nuclear explosion in Shaft 1352. The first two took place at the NTS in volcanic tuffs and the third occurred at the Semipalatinsk Test Site (STS) in granite. Table 1 summarizes the explosion information and the aftershock reference.

Hamilton (1972b) measured the magnitude distribution of BENHAM aftershocks and found a $b$ value of 1.4, but noted this may be due to underestimation of larger aftershocks, in which case a value closer to 1.0 is more appropriate. STAUDER (1971) performed a more thorough study of BENHAM aftershocks and found a $b$ value of 1.02. This is the value we will use to characterize the sequence. Hamilton and Healy (1969) plot the number of aftershocks per day as a function of days after the explosion. The decay has power-law behavior until the 15th day, when a $M_{\mathrm{L}} 3.7$ earthquake occurred nearby that caused an increase in measured aftershocks (Hamilton 1972b). Therefore, we only calculate $p$ and $K$ from the first 15 days of observation (Fig. 1). BENHAM was a $M_{\mathrm{L}} 6.3$ (HAMILTON 1969) and the minimum magnitude measured in the Hamilton (1972b) study was $M_{\mathrm{L}} 1.3$, which gives a $\Delta M=5$. Hamilton et al. (1972a) also plot the number of aftershocks per $\mathrm{km}$ as a function of distance from the explosion. We use this information to calculate $n$ (Fig. 2).

JARPE et al. (1994) presented the magnitude distribution of the NPE, which we measured to find a $b$ value of 1.10 . Since a count of aftershocks per day was made at only a few times, JARPE (1994) combined 
Table 1

Explosion aftershock data set information

\begin{tabular}{llllrrr}
\hline Shot & Date & Site/location & Type & Yield (kt) & Depth (m) & References \\
\hline BENHAM & 19-Dec-68 & NTS/Pahute Mesa & Welded tuff & 1,150 & 1,402 & HAMILTON (1972b) \\
NPE & 22-Sep-93 & NTS/Rainier Mesa & Tuff & 1 & 390 & JARPE (1994) \\
Shaft 1352 & 8-Jul-89 & STS/Balapan & Granite & 35 & 550 & ADUSHKIN (1995) \\
\hline
\end{tabular}

NPE Non-Proliferation Experiment, NTS Nevada Test Site, STS Semipalatinsk Test Site

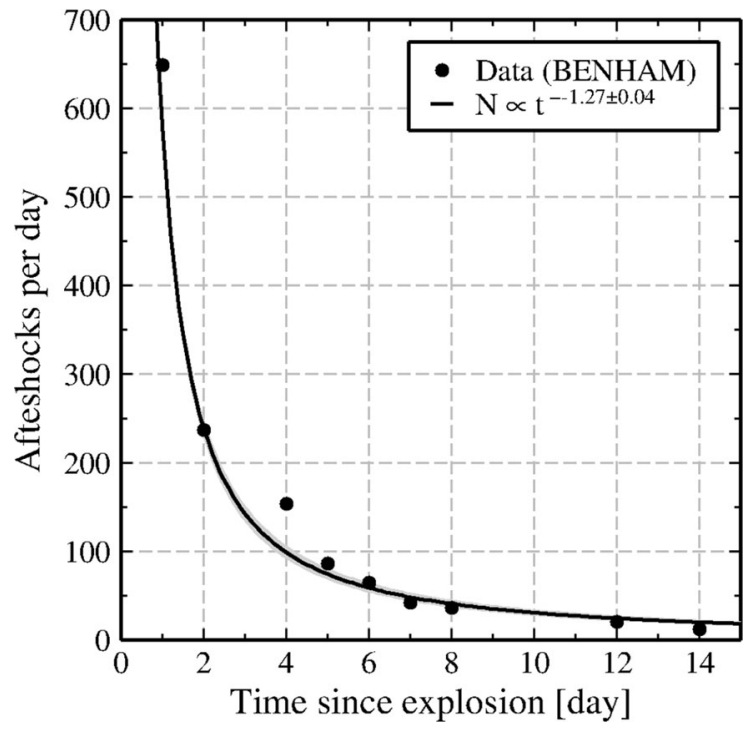

Figure 1

Aftershock rate for the explosion, BENHAM (HAMILTON 1969). The shaded area is the formal error in the model

the measurements with other explosion aftershock sequences in similar geology. We use this combined data set to measure $p$ and $K$ (Fig. 3); however, since there are so few measurements, the error is large. JARPE (1994) report that the NPE was a $M_{\mathrm{L}} 4.0$ and the minimum magnitude that was measured was $M_{\mathrm{L}}-0.5$, which gives a $\Delta M=4.5$. Explosion aftershocks were not located in any of these studies, so a determination of $n$ cannot be made.

Adushrin and SpIVAK (1995) report on several explosion aftershock studies at STS and they analyze in detail the Shaft 1352 explosion. The $b$ value inferred from their plot of aftershock amplitude distribution is 1.00 . The $p$ and $K$ values were extracted from the plot of aftershocks per hour, by ADUSHKIN (1995) (Fig. 4). Adushrin (1995) did not report the minimum magnitude measured in the aftershock

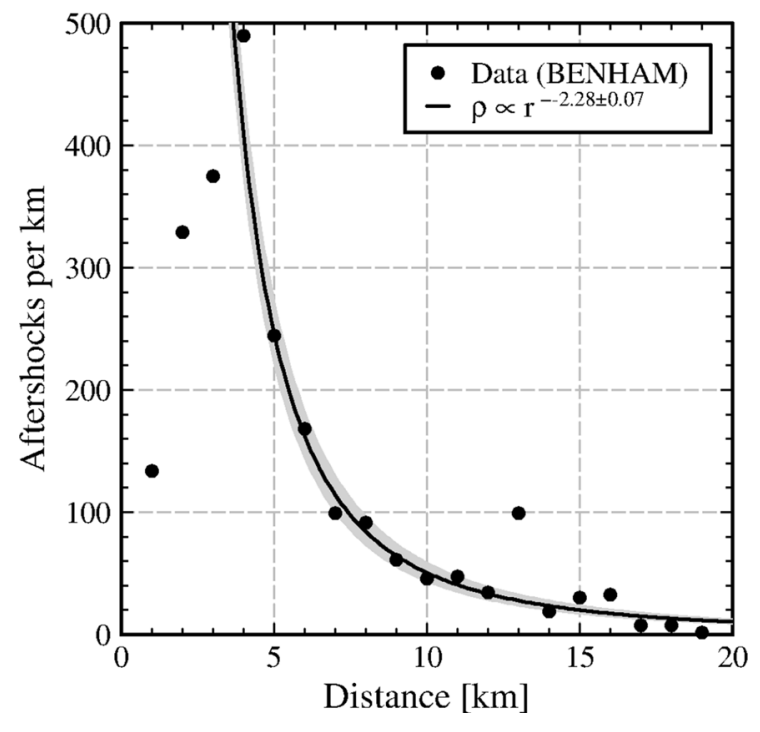

Figure 2

Aftershock linear density for the explosion, BENHAM (HAMILTON 1972b). The shaded area is the formal error in the model for distance $>750 \mathrm{~m}$

study, but rather reported peak velocities for each event in order to infer a $b$ value. We therefore used a magnitude (MPV) equation appropriate for local-toregional distance $P$ waves recorded in the region, $\mathrm{MPV}=\log _{10}(A / T)+\sigma(r)$ (A. Belyashov, personal communication), where $A$ is the displacement, $T$ is the period of the measurement, and $\sigma$ is the attenuation correction that is a function of distance $r$. The smallest distance for which there is a correction is $10 \mathrm{~km}$, but the study at Shaft 1352 measured aftershocks at a distance of $\sim 1 \mathrm{~km}$, so we extrapolate the correction to that distance and we estimate that the aftershocks had a period of measurement of $\sim 0.1 \mathrm{~s}$. We also convert the reported smallest velocity measured in the study, $0.4 \mu \mathrm{m} / \mathrm{s}$, to a displacement by dividing by $2 \pi$ under the harmonic assumption. The minimum 


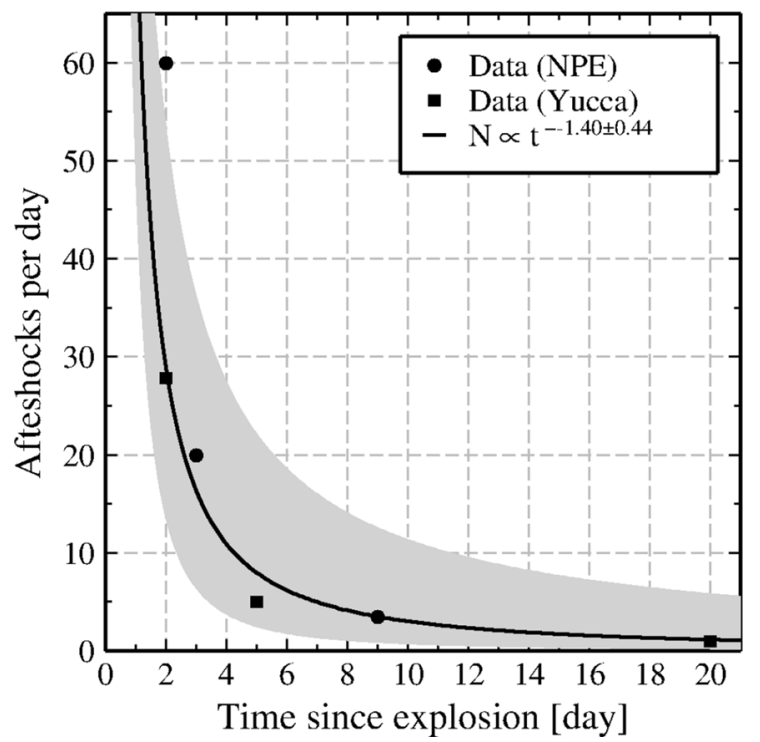

Figure 3

Aftershock rate for the explosion, NPE (circles), and others in similar geology (squares) (JARPE 1994). The shaded area is the formal error in the model

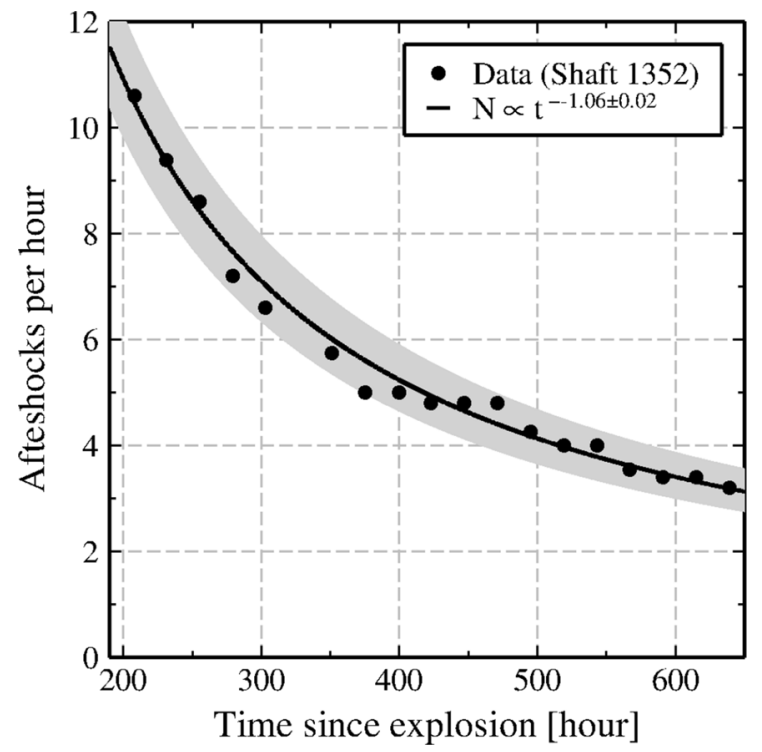

Figure 4

Aftershock rate for the Shaft 1352 explosion (ADUSHKIN 1995). The shaded area is the formal error in the model

magnitude in the study is $M-0.3$ and the explosion magnitude was 5.6 (KHALtURIN et al. 2001). AdUSHKIN (1995) also plot the number of aftershocks as a function of distance from the explosion. We use this information to calculate $n$ (Fig. 5).

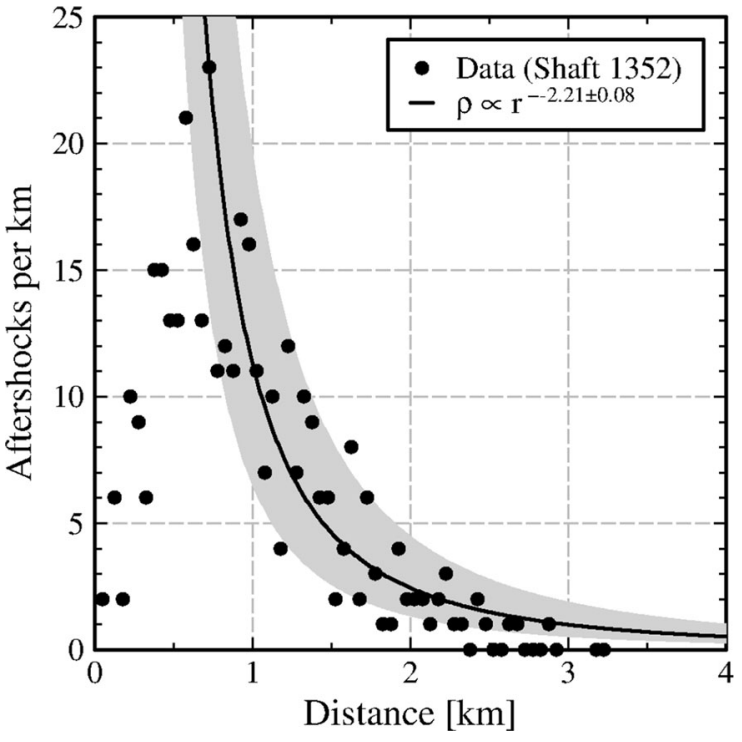

Figure 5

Aftershock linear density for the Shaft 1352 explosion (ADUSHKIN 1995). The shaded area is the formal error in the model

Since BENHAM occurred on Pahute Mesa in more competent, higher-strength volcanics than the NPE on Rainier Mesa, we will refer to the models from these two sequences as NTS-hard and NTS-soft, respectively. The explosion in Shaft 1352 occurred in granite at Balapan, and the model from this sequence will be named STS-hard. Table 2 summarizes the model parameters for each aftershock data set.

Using the parameters given in Table 2, we define a family of three aftershock models that span a range of geologic media, NTS-hard (BENHAM), NTS-soft (NPE), and STS-hard (Shaft 1352). Figure 6 gives the aftershock rate of events with magnitudes greater than five units less than the explosion $(\Delta M=5)$ for each of the explosion models, and for general comparison, an earthquake aftershock model used by the US Geological Survey, SoCal (Gerstenberger et al. 2004). The earthquake model has the greatest rate, followed by the explosion models ordered by decreasing medium strength or competency, STS-hard, NTS-hard, and NTS-soft. We can manipulate the event probability to estimate the probability of at least one aftershock larger than a given magnitude (1-probability of no aftershocks). Figure 7 shows these probabilities for each model as a function of time after the explosion for $\Delta M=2$. We can also 
Table 2

Aftershock model parameters

\begin{tabular}{lllllllll}
\hline Shot & Name & $M_{\mathrm{m}}$ & $M_{\min }$ & $\log _{10}(K)$ & $a$ & $p$ & $b$ \\
\hline BENHAM & NTS-hard & 6.3 & 1.3 & 2.76 & -2.69 & 1.36 & 1.02 \\
NPE & NTS-soft & 4.0 & -0.5 & 2.00 & -3.40 & 1.53 & 1.10 \\
Shaft 1352 & STS-hard & 5.6 & -0.3 & 3.38 & -2.47 & 1.06 & 1.00 \\
a & SoCal & - & - & - & -1.67 & 1.08 & 0.91 & 2.21 \\
\hline
\end{tabular}

${ }^{\text {a }}$ SoCal model is from an analysis of Southern California earthquakes (GeRSTENBERGER 2004)

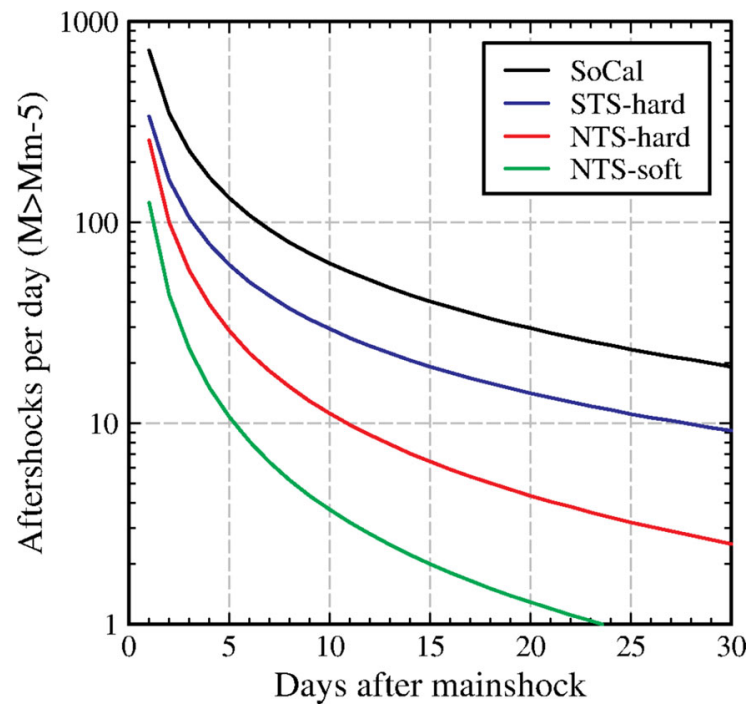

Figure 6

Aftershock rate (events/day) for the three explosion models (color) and an earthquake model (black) for events with magnitude greater than the mainshock magnitude $\left(M_{\mathrm{m}}\right)$ minus 5

manipulate the event probability to estimate the cumulative number of expected aftershocks given a probability and observation time after the explosion. Figure 8 shows these numbers for each model where recording starts 1 day, 1 week, and 1 month after the explosion occurs. Note that only three events are predicted in a 3-day observation period for the NTSsoft model where observation begins 1 month after the explosion (Fig. 8c).

\section{Application}

If we combine the explosion aftershock model with a network geometry and station detection level, we can predict, with a given probability, the

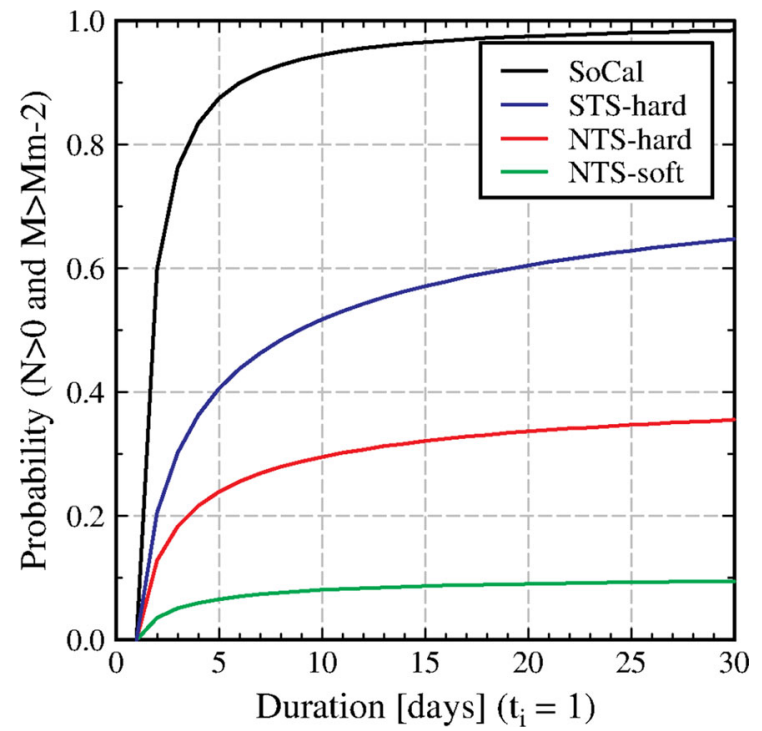

Figure 7

Probability of at least one aftershock with magnitude greater than two less than the mainshock magnitude where the duration begins $\left(t_{i}\right) 1$ day after the mainshock time $\left(t_{0}\right)$ for the three explosion models (color) and an earthquake model (black)

observation of an explosion aftershock at a given time since the explosion. Details of the mini-arrays and processing used in a Seismic Aftershock Monitoring System (SAMS) deployment are given in SICK and Joswig (2014). Figure 9 gives the magnitude of detection $\left(M_{\mathrm{D}}\right)$ curve for the SAMS, as determined from data for a field experiment in Finland in 2009 (DE09) in competent rock, fit with a second order polynomial on log-distance. We randomly design a potential OSI deployment geometry and calculate the predicted number of aftershocks detected at each station with a probability of 0.90 for a given day after the explosion. Figure 10 shows the results for 1 week 


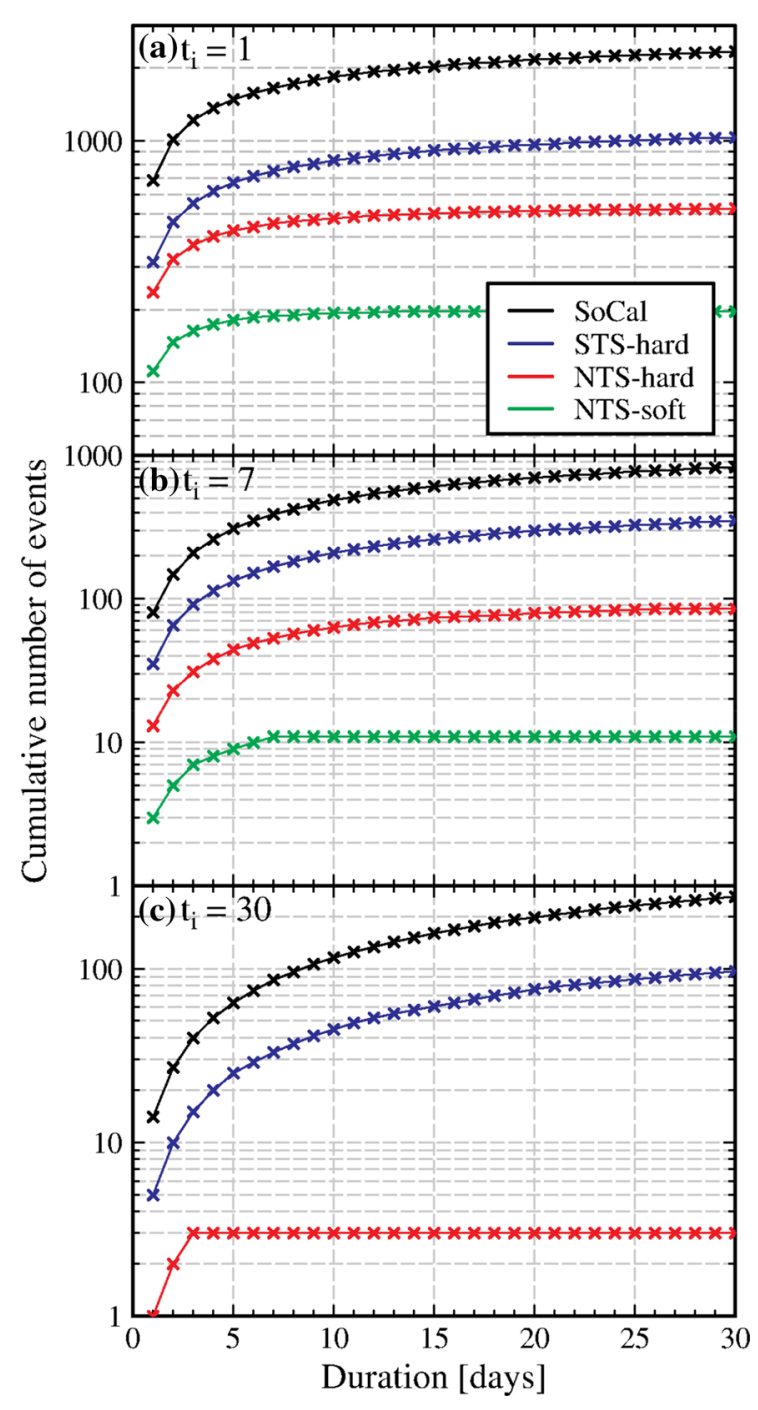

Figure 8

Expected cumulative number of events with magnitude greater than five less than the mainshock magnitude at a probability of 0.90 for the NTS-hard model where recording begins 1 day (a), 1 week (b), and 1 month (c) after the mainshock for the three explosion models (color) and an earthquake model (black)

and 1 month after an M5 and M4 explosion-triggering event.

\section{Discussion}

The $b$ value is near one for all models, which is similar to earthquake studies, though there is some suggestion that weaker source material increases the $b$ value, but with such small studies, this is difficult to

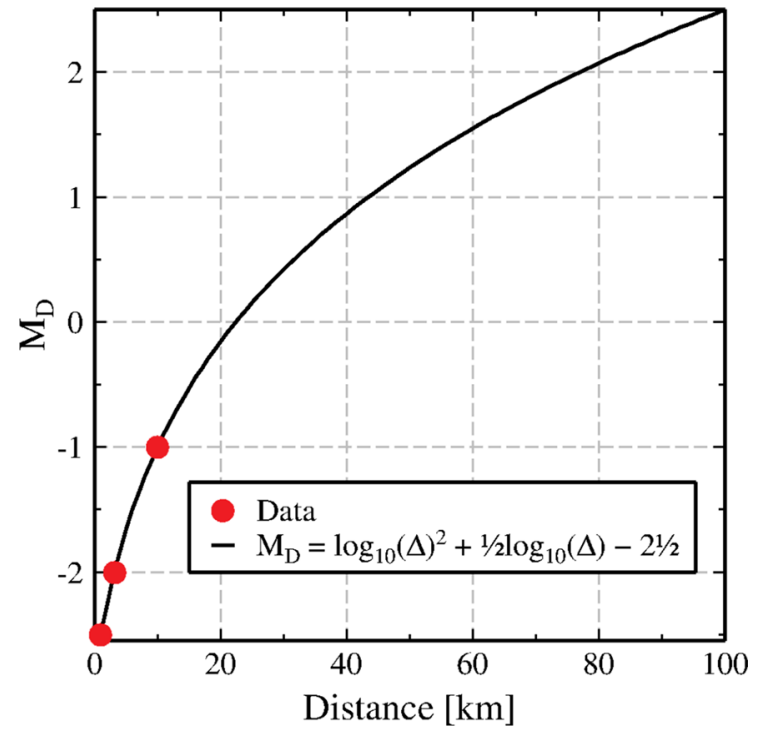

Figure 9

Magnitude of detection as a function of distance from the event. The data (red circles) are fit with a simple second order polynomial on log-distance (black line)

demonstrate. An apparent relationship to source medium strength is the decrease of $p$ value and increase in $a$ value, so that explosion aftershocks in competent rock are more abundant for a longer duration than aftershocks in weaker media. PHILLIPS et al. (1999) analyzed the aftershock sequence of a controlled mine collapse and found a $p$ value $=1.3$, similar to the NTS-hard model. KGARUME et al. (2010) found values close to 1 for both the $p$ and $b$ values in gold mine tremor aftershocks, where the distance decay parameter suggested dynamic triggering. The distance decay ( $\gamma$ value) in explosion aftershocks is similar in both the 'hard' models and greater than some earthquake models, suggesting static stress as the dominant transfer mechanism, which is consistent with the conclusions of PARSONS and Velasco (2009). Also, there seems to be an offset in distance from the detonation point, where the power-law behavior begins. This offset is greater than the cavity radius, and may be related to poor locations from simple velocity models that don't account for damage around the cavity.

The detections per station shown in Fig. 10 can be used in planning and interpretation of seismic data from an OSI for any given station configuration. These maps are very dependent on the magnitude of 


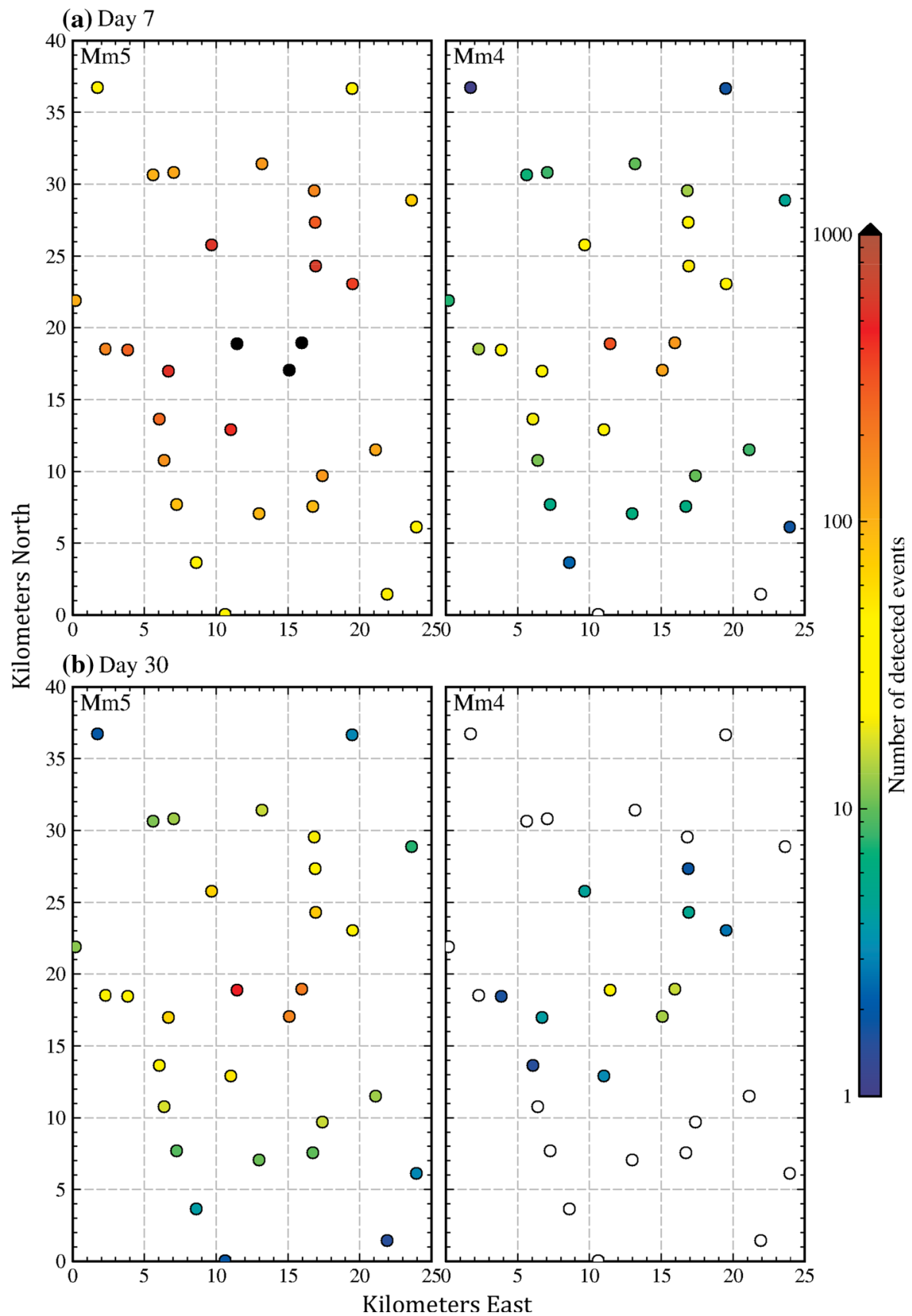

Figure 10

Number of detected events over a 25 by $40 \mathrm{~km}$ area at a given station (circle) for a explosion at the center of the deployment (12/1/2, 20) with magnitude 5 (Mm5, left panel) and $4(\mathrm{Mm} 4$, right panel), 1 week (a) and 1 month (b) after the explosion. White circles are zero detections

detection relationship (as given in Fig. 9), where stations close to the explosion epicenter can detect more than a thousand events in a day even days after the large explosion. This is due to the very low detection threshold of the SAMS, so that for a explosion magnitude of 5 (left panel, Fig. 10), stations $10 \mathrm{~km}$ away from the epicenter detect events according to a rate equation where $\Delta M=6$. This effect can be seen when comparing with a explosion magnitude 4 event (right panel, Fig. 10), where the number of detections 
is an order of magnitude less. These predictive models could be improved with more data to define the detection threshold and its error.

The predictive power of the explosion aftershock models could be used in the planning and deployment of the SAMS in a potential OSI as part of the Comprehensive Test Ban Treaty. The planned deployment could be evaluated for a predicted number of aftershocks detected, and this could guide the OSI team in its use of resources. Ideally, maps like those shown in Fig. 10 would have an additional layer of seismic noise to help in determining the true detection threshold at a site (SicK et al. 2013).

\section{Conclusions}

The spatial and temporal distribution of aftershocks due to an explosion can be described with the aftershock rate and distance decay models used for earthquake aftershocks. The parameters for the model are dependent on the source medium, and we define three parameterizations based on aftershock records from explosions in hard rock at STS and NTS, as well as less competent rock at NTS. The log-ratio of the number of large to small events ( $b$ value) for each model is approximately one, and the decay rate ( $p$ value) decreases and productivity ( $a$ value) increases with source medium strength.

The models are applied to a fictitious site inspection scenario and combined with station detection thresholds and site geometry to predict the number of events detected for a given explosion magnitude and observation day. These predictions can be used as tools to aid in the deployment and analysis of Seismic Aftershock Monitoring System data during an on-site inspection scenario.

\section{Acknowledgments}

The authors are grateful for two anonymous reviews and a review by the Associate Editor, Anton Dainty. His efforts in producing the highest quality articles regarding explosion monitoring, and seismic analyses in general have benefitted the entire community and will be greatly missed.The authors are grateful for support from the US Department of Energy, National Nuclear Security Administration, Nonproliferation and International Security and the Comprehensive Test Ban Treaty Organization Preparatory Commission, Conference on Science and Technology. This research was performed in part under the auspices of the US Department of Energy by the Lawrence Livermore National Laboratory under contract number DEAC52-07NA27344; Information Management release number LLNL-JRNL-652465.

Open Access This article is distributed under the terms of the Creative Commons Attribution License which permits any use, distribution, and reproduction in any medium, provided the original author(s) and the source are credited.

\section{REFERENCES}

Adushrin, V., and A. Spivak (1995). Aftershock of Underground Nuclear Explosion, in Earthquakes Induced by Underground Nuclear Explosions, eds. R. Console and A. Nikolaev, SpringerVerlag, Berlin, 35-49.

Felzer, K. R. and E. E. Brodsky (2006). Decay of aftershock density with distance indicates triggering by dynamic stresses, Nature, 441, 735-738.

FoRD, S. R., and W. R. WALTER (2010). Aftershock Characteristics as a Means of Discriminating Explosions from Earthquakes, Bull. Seis. Soc. Amer., 100, 364-376, doi:10.1785/0120080349.

Gerstenberger, M. C., S. Wiemer, And L. M. Jones (2004). Realtime forecasts of tomorrow's earthquakes in California: a new mapping tool, US Geological Survey Open-File Report 2004-1390.

Hamilton, R. M. and J. H. Healy (1969). Aftershocks of the BENHAM nuclear explosion, Bull. Seismol. Soc. Amer., 59, 2271-2281.

Hamilton, R. M., F. A. Mckeown, and J. H. Healy (1972a). Seismic activity and faulting associated with a large underground nuclear explosion, Science, 166, 601-604.

Hamilton, R.M., B.E. Smith, F.G. Fischer, and P.J. Papanek (1972b). Earthquakes caused by underground nuclear explosions on Pahute Mesa, Nevada Test Site, Bull. Seismol. Soc. Amer., 62, 1319-41.

JARPE, S., P. Goldstein, and J. J. ZucCA (1994). Comparison of the non-proliferation event aftershocks with other Nevada Test Site events, UCRL-JC-117754, in Non-proliferation Experiement Symposium, Rockville, Maryland, 19-21 April 1994.

Kgarume, T. E., S. M. Spottiswoode, R. J. Durrheim (2010). Statistical properties of mine tremor aftershocks, Pure Appl. Geophys., 167, 107-117, doi:10.1007/s00024-009-0004-5.

Khalturin, V.I., T.G.Rautian, and P.G.Richards. (2001) A study of small magnitude seismic events during 1961-1989 on and near the Semipalatinsk Test Site, Kazakhstan, Pure Appl. Geophys., 158, 143-171.

Kitov, I. O., and O. P. Kuznetov. (1990) Energy released in aftershock sequence of explosion, Doklady Akademii Nauk SSSR, 315, 839-842. 
Parsons, T., and A. A. Velasco (2009). On near-source earthquake triggering, J. Geophys. Res., 114(B10307), doi:10.1029/ 2008JB006277.

Phillips, W. S., D. C. Pearson, X. Yang, and B. W. Stump (1999). Aftershocks of an explosively induced mine collapse at White Pine, Michigan, Bull. Seis. Soc. Amer., 89(6) 1575-1590.

REASENBERG, P. A., and L. M. Jones (1989). Earthquake hazard after a mainshock in California, Science, 243, 1173-1176.

Reasenberg, P. A., and L. M. Jones (1994). Earthquake aftershocks - Update, Science, 265, 1251-1252.

Sick, B., N. Gestermann, and M. Joswig (2013), Seismic aftershock monitoring network optimization based on detection threshold estimation from background noise measurement, Science and Technology Conference, Vienna.

Sick, B., M. Walter, M. Joswig (2014). Visual Event Screening of Continuous Seismic Data by Supersonograms, Recent Advances in Nuclear Explosion Monitoring Vol. 2, Pure Appl. Geophys., 171, 549-559, doi:10.1007/s00024-012-0 618-X.

STAUDER, W. (1971). Smaller aftershock of Benham nuclear explosion, Bull. Seismol. Soc. Amer., 61, 417-428.

WIEMER, S. (2000). Introducing probabilistic aftershock hazard mapping, Geophysical Research Letters, 27(20), 3405-3408.

(Received May 1, 2014, revised January 13, 2015, accepted January 16, 2015, Published online February 14, 2015) 